\title{
Different body parts' fat mass and corrected QT interval on the electrocardiogram: The Fasa PERSIAN Cohort Study
}

\author{
Mohammad Hosein Yazdanpanah ${ }^{1,2}$, Ehsan Bahramali ${ }^{3}$, Mohammad Mehdi Naghizadeh², Mojtaba Farjam²*,
} Maryam Mobasheri ${ }^{1}$ and Shiva Dadvand ${ }^{1}$

\begin{abstract}
Background: Previous studies suggested that obesity and fat mass are associated with QT interval prolongation, but the role of different body parts' fat mass is unclear. The associations between total and regional fat mass (FM) and corrected QT interval (QTC) were investigated for the first time in this study.

Methods: In this sub-analysis of Fasa PERSIAN cohort Study data, 3217 subjects aged 35-70 entered our study. Body fat mass was assessed by bioelectrical impedance analysis and QTc interval calculated by the QT interval measured by Cardiax ${ }^{\circledR}$ software from ECGs and Bazett's formula. Uni- and multi-variable linear and logistic regression was performed in IBM SPSS Statistics V23.

Results: In males, the fat mass to fat-free mass (FM/FFM) ratio in the trunk, arms, total body, and legs were significantly higher in the prolonged QTc group (QTc $>450 \mathrm{~ms})$. Trunk $(B=0.148)$, total $(B=0.137)$, arms $(B=0.124)$, legs $(B=0.107)$ fat mass index (FMI) showed significant positive relationship with continuous $Q T C$ ( $P$-value $<0.001)$. Also, just the fat-free mass index of legs had significant positive associations with QTc interval (P-value $<0.05$ ). Surprisingly, in females, the mean of FM/FFM ratio in trunk and legs in the normal QTc group had higher values than the prolonged QTc group (QTc > $470 \mathrm{~ms}$ ). Also, none of the body composition variables had a significant correlation with continuous QTC.

Conclusion: Our study suggested that FMl ratios in the trunk, total body, arms, and legs were positively associated with QTc interval in males, respectively, from a higher to a lower beta-coefficient. Such associations were not seen in females. Our study implies that body fat mass may be an independent risk factor for higher QTc interval and, consequently, more cardiovascular events that should be investigated.
\end{abstract}

Keywords: Fat mass, Fat mass to fat-free mass (FM/FFM) ratio, QT interval, QTC prolongation, Cardio-metabolic, Obesity, Electrocardiogram, Iran, Load-capacity model

*Correspondence: farjam.md@gmail.com; mfarjam@fums.ac.ir

${ }^{2}$ Noncommunicable Diseases Research Center, Fasa University of Medical

Sciences, Fasa, Iran

Full list of author information is available at the end of the article

\section{Introduction}

Total depolarization and repolarization times of cardiomyocytes are reflected in the QT interval in the surface electrocardiogram (ECG). Thus, any alteration in the QT interval can indicate abnormalities in the two phases of the cardiomyocyte electrical cycle. Any QT prolongation is primarily secondary to a prolonged repolarization period, and it may be congenital or acquired. Some of the 
known and suggested risk factors of prolonged QT are age [1], female gender [2], smoking [3], hyperlipidemia [4] and a history of prior cardiovascular diseases [5], metabolic syndrome [6], and comorbidities like diabetes, renal failure, thyroid disturbances [7], and liver failure [8]. Electrolytes like potassium [9] and calcium [7] have immediate and long-term effects on the QT interval; however, it is more of a concern in acute settings. Also, there is a long list of drugs that affect QT, some of which can produce arrhythmia [10].

One of the most prevalent cardiovascular risk factors which can affect QT interval, directly and indirectly, is obesity. Increasing body mass index (BMI) can alter the heart rate and, thus, the corrected QT [2, 3]. Obesity is also associated with other comorbidities and cardiovascular diseases (CVD) risk factors, including diabetes, hypertension, and hyperlipidemia. Obesity can lead to increased morbidity and mortality due to CVD as important NCDs among the Iranian population [11-13]. A previous study in an Iranian population showed that central adiposity is a significant determinant in CVD risk [14]. Also, in the Framingham study, obesity was one of the significant causes of mortality due to heart disease [15].

Compelling evidence indicates that the QT interval is longer in overweight or obese individuals than in the average population [16]. Interestingly, after weight loss, either through diet and exercise or through surgery, the QT interval shortens $[16,17]$. Making it more quantitative, Carella et al. showed a 5-ms increase in QT interval by every $50 \%$ increase in fat mass percentage measured by hydrodensitometry. The health effect of fat mass is not about the fat volume itself but metabolic, neuroendocrine, and inflammatory changes linked to fat mass and its regional depots. Some of the suggested fat mass effect mechanisms include impaired fasting glucose [18], hyperinsulinemia and insulin resistance [19], HR-variability, and SNS activity [20].

Longer QT intervals have historically been believed to be a risk factor for developing arrhythmia in normal and diseased hearts [21]. The QT interval has served as a prognostic factor in specific subgroups of patients, including those with ischemic heart disease or heart failure. Other measures related to it, like QT dispersion, have received much attention as predictors of sudden death in these patient populations [22]. The heterogeneity of cardiomyocyte electrical activity, reflected in surface ECG as QT interval, explains why QT interval can discriminate an arrhythmia-prone heart and consequently serves as a predictive tool [23].

Determining body composition and distribution of fat in body parts has been made possible using various devices that introduce small electrical currents through different routes in the corpus and measures the electrical impedance accordingly. Body composition as a reliable way for distinguishing the fat disturbances in various body parts was used in our study to seek which body part's fat mass is associated with corrected QT (QTc) interval in an Iranian population.

\section{Methods \\ Study population}

In this cross-sectional study, we used collected data of Fasa Cohort Study, a branch of Prospective Epidemiological Research Studies in IrAN (PERSIAN) [24]. This study is a prospective population-based cohort to evaluate non-communicable diseases (NCDs) in participants aged 35-70 with a 15-year follow-up. Before registration in Fasa Cohort Study, informed consent is obtained from each participant. All subjects with available data of both body composition and electrocardiograms $(n=3311)$ entered our study. Nighty-four subjects (2.83\%) were excluded due to lack of data of ECG and Body composition, non-sinus rhythm, wide QRS complex (QRS duration $>120 \mathrm{~ms}$ ), which can falsely increase QT interval. Eventually, 3217 subjects (1415 male, 1802 female) remained in the study. Demographic data, including age, sex was recorded in a questionnaire. Also, the current status of smoking has been questioned: "Do you smoke cigarettes? Yes or no". NCDs' history, such as diabetes and hypertension, and coronary heart disease (CHD), have been questioned and recorded. Diabetes was determined from self-reported history, use of oral hypoglycemics, or insulins. Coronary heart disease was determined by the self-reported history of coronary artery bypass graft surgery, coronary angioplasty, myocardial infarction, angina, or ECG evidence of myocardial infarction by identifying major Q-wave. Medication taken within two weeks was asked to be brought for registration at interview time for maximum precision. CredibleMeds, as an American organization, provides lists of drugs associated with QTcprolongation, which is continuously updated by their review team based on new information. We used a list of including drugs with a known risk for TdP and medications with a possible risk for TdP, which counts 187 cardiac and non-cardiac drugs in total [25].

\section{Measurements}

For serum total and high-density lipoprotein cholesterol (TC and HDL) assessment, venous blood samples were taken in a 10-14 $\mathrm{h}$ fasting condition. The sampling was performed by trained personnel and transported to the laboratory in standard condition. TC and HDL measures were assayed using the Mindray BS380 autoanalyzer (Mindray Medical International, Shenzhen, China) for the biochemical tests. A stadiometer measured height 
with an accuracy of $0.1 \mathrm{~cm}$, and a digital scale measured weight with an accuracy of $0.1 \mathrm{~kg}$. Body mass index (BMI) was calculated using a value divided by the square of height $\left(\mathrm{kg} / \mathrm{m}^{2}\right)$. A nurse was in charge of taking resting blood pressure. Systolic and diastolic blood consists of 2 measurements in the seating position in $15 \mathrm{~min}$ and is reported by an average pressure in $\mathrm{mmHg}$.

\section{Body composition}

Bioelectric impedance analysis (BIA) was used for measuring body composition using the Tanita BC-418 MA Segmental Body Composition Analyzer (Tanita, Japan). This device is a single-frequency BIA device with eight polar electrodes and a single-point load cell weighing system in the scale platform that can provide separate body mass readings for various body segments, including the right arm, left arm, trunk, right leg, and left leg. The impedance across the subject's tissues is determined with receiver electrodes after a predefined signal is passed through injector electrodes. All measurements are performed at $50 \mathrm{kHz}$ with a steady current of $0.8 \mathrm{~mA}$ sine wave. FM percent is calculated using an algorithm that takes into account impedance, age, and height. Body composition assessment was reported by arm fat mass (AFM), arms fat-free mass (AFFM), legs fat mass (LFM), legs fat-free mass (LFFM), trunk fat mass (TFM), truncal fat-free mass (TFFM), total fat mass (FM) and total fat-free mass (FFM). Fat mass percentages in total and regional depots were also calculated. The fat(-free) mass index (FMI) was calculated using fat(-free) mass (total or regional depots) in $\mathrm{Kg}$ divided by the square of height in $\mathrm{m}(\mathrm{kg} / \mathrm{m} 2)$. Also, the fat mass to fat-free mass ratio (FM/ FFM) has been calculated for total and different body regions. These ratios are representing the load-capacity model, following Siervo et al. [26]. FM has a negative influence on physiological function, while FFM has a beneficial effect in this model.

\section{Electrocardiogram}

Each participant had 12-lead electrocardiograms (ECG) with a $2000 \mathrm{~Hz}$ sampling rate and $0.04 \mu \mathrm{V} /$ bit (24-bit resolution). ECGs were recorded by trained personnel using a computer-based device $\left(\operatorname{Cardiax}^{\circledR}\right)$ [27], and the recording was performed on shaved precordium in the supine and postprandial state for the best possible results. Heart rates and QT intervals (Lead II) of all ECGs were reported automatically by Cardiax software (version 3.50.2, International Medical Equipment Developing Co. Ltd., Budapest, Hungary) and exported to central data software. Also, QTc was calculated by Bazett's formula $\left(\mathrm{QTc}=\mathrm{QT} /(\mathrm{RR})^{1 / 2}\right)$ [28]; Bazett's formula was used due to widespread use in clinical situations and having the standardized clinical cut-offs. Based on clinical standards, QTc $<450 \mathrm{~ms}$ for males and QTc $<470 \mathrm{~ms}$ for females was considered Normal QTc, higher QTc than these two cut-points considered as prolonged QTc in both genders $[29,30]$.

\section{Statistical analyses}

All variables were reported as Mean \pm standard deviation (SD), and number (Percentage \%). There was not any missing data among the raw data. For comparison between the two groups, we used an independent-samples t-test. To determine the correlation between body composition and continuous QTc, linear regression was used, also with dichotomous QTc (Normal and Prolonged), logistic regression was used. The regression standardized beta coefficient (B) with its P-value in the first analysis and the OR and 95\% Confidence intervals $(95 \% \mathrm{CI})$ with its $\mathrm{p}$-value in the last analysis were reported. About regression analysis were adjusted by conventional CVD risk factors, including age (years), smoking status (yes $=1)$, total cholesterol $(\mathrm{mg} / \mathrm{dL})$, highdensity lipoprotein $(\mathrm{mg} / \mathrm{dL})$, and blood pressure $(\mathrm{mmHg})$, history of CHD (yes $=1$ ), diabetes (yes $=1$ ), and selected medication as drugs known for prolonging QT interval (consume $=1$ ). More details of drugs were mentioned above25. Also, 5th, 15th, 25th, 50th, 75th, 85th, and 95th percentiles for FM/FFM ratio have been calculated in both genders. Significant P-value was considered at the P-value level $<0.05$, and all analysis was performed using IBM SPSS Statistics, version 23 (IBM Corp., Armonk, N.Y., USA). Also, for graphs, we used Prism version 8.00 (GraphPad Software, La Jolla California, USA).

\section{Results}

In a total of 3217 participants, the mean age was $47.39 \pm 9.23$ years in men and $48.12 \pm 9.55$ years in women. The mean BMI was $23.96 \pm 4.50 \mathrm{~kg} / \mathrm{m}^{2}$ in men and $27.03 \pm 4.97 \mathrm{~kg} / \mathrm{m}^{2}$ in women. There were $794(56.1 \%)$ male subjects, and $68(3.8 \%)$ of the females reported active smoking. In males, 104(7.3\%) and 302(16.8\%) reported diabetes in females. The mean of QT interval before and after correction respectively in men was $403.33 \pm 37.72 \mathrm{~ms}$ and $418.00 \pm 32.85 \mathrm{~ms}$, and in women was $396.61 \pm 42.38 \mathrm{~ms}$ and $438.65 \pm 39.77 \mathrm{~ms}$. The difference between males and females in QT and corrected QT intervals was statistically significant $(\mathrm{P}$-value $<0.001$ ). Other variables mean and SD, number (\%) according to gender reported in Table 1.

Table 2 shows the means of total and regional body composition data and BMI in total, normal QTc, and prolonged QTc interval groups according to gender. Total FMI was $4.97 \pm 2.55 \mathrm{~kg} / \mathrm{m}^{2}$ and $9.56 \pm 3.45 \mathrm{~kg} / \mathrm{m}^{2}$ in the total male and female population, respectively. Total FMI and truncal FMI were higher in males with prolonged 
Table 1 Baseline characteristic of subjects according to gender

\begin{tabular}{|c|c|c|c|}
\hline Variables & Male $(n=1415)$ & Female $(n=1802)$ & P-value \\
\hline Age (years) & $47.39 \pm 9.23$ & $48.12 \pm 9.55$ & $0.030^{\mathrm{a}}$ \\
\hline BMI $\left(\mathrm{kg} / \mathrm{m}^{2}\right)$ & $24.19 \pm 4.41$ & $26.86 \pm 4.81$ & $<0.001^{a}$ \\
\hline $\mathrm{SBP}(\mathrm{mmHg})$ & $108.31 \pm 16.99$ & $109.35 \pm 18.56$ & $0.097^{a}$ \\
\hline $\mathrm{DBP}(\mathrm{mmHg})$ & $72.43 \pm 11.85$ & $72.46 \pm 11.94$ & $0.933^{\mathrm{a}}$ \\
\hline $\mathrm{TC}(\mathrm{mg} / \mathrm{dL})$ & $178.13 \pm 38.64$ & $189.01 \pm 39.25$ & $<0.001^{\mathrm{a}}$ \\
\hline $\mathrm{HDL}(\mathrm{mg} / \mathrm{dL})$ & $43.49 \pm 9.16$ & $49.62 \pm 10.31$ & $<0.001^{\mathrm{a}}$ \\
\hline Current smoker & $794(56.1)$ & $68(3.8)$ & $<0.001^{b}$ \\
\hline $\begin{array}{l}\text { Coronary heart } \\
\text { disease }\end{array}$ & $80(5.7)$ & $206(11.4)$ & $<0.001^{b}$ \\
\hline Diabetes mellitus & $104(7.3)$ & $302(16.8)$ & $<0.001^{b}$ \\
\hline Hypertension & $141(10.0)$ & $497(27.6)$ & $<0.001^{\mathrm{b}}$ \\
\hline Selected medications & $965(68.2)$ & $1195(66.3)$ & $0.259^{b}$ \\
\hline Heart rate (bpm) & $65.61 \pm 10.79$ & $74.51 \pm 11.76$ & $<0.001^{\mathrm{a}}$ \\
\hline QRS duration (ms) & $105.31 \pm 9.23$ & $90.87 \pm 6.35$ & $<0.001^{\mathrm{a}}$ \\
\hline QT interval (ms) & $403.33 \pm 37.72$ & $396.61 \pm 42.38$ & $<0.001^{\mathrm{a}}$ \\
\hline $\begin{array}{l}\text { Bazett's QTc interval } \\
\text { (ms) }\end{array}$ & $418.53 \pm 32.85$ & $438.65 \pm 39.77$ & $<0.001^{\mathrm{a}}$ \\
\hline $\begin{array}{l}\text { Fridericia's QTc inter- } \\
\text { val (ms) }\end{array}$ & $413.12 \pm 30.93$ & $423.92 \pm 37.95$ & $<0.001^{\mathrm{a}}$ \\
\hline
\end{tabular}

Data presented as mean \pm standard deviation, number (frequency)

$\mathrm{BMI}=$ body mass index, $\mathrm{SBP}=$ systolic blood pressure, $\mathrm{DBP}=$ Diastolic blood pressure, $\mathrm{TC}=$ Total cholesterol, $\mathrm{HDL}=$ High density lipoprotein, $\mathrm{QTC}=$ corrected QT interval

$\mathrm{P}$-value reported as the result of aindependent-samples t-test and ${ }^{\mathrm{b}} \mathrm{Chi}$-square. Statistically significant P-values are bolded ( $P$-value $<0.05$ )

QTc and showed a significant difference between normal and prolonged QT groups (P-value $<0.05)$, while it was higher in females with normal QTc, it did not show any significant difference between groups. Both fat percentage and FM/FFM ratio in total, arms, and trunk showed a significant difference between QT interval groups in males. Also, in females, truncal fat percentage and FM/ FFM ratio showed a significant difference between QT interval groups, but their mean was higher in the normal QT group than in a prolonged QT group $(\mathrm{P}$-value $<0.05)$.

In males, AFM, TFM, and Total FM were significantly higher in the prolonged QTc group compared to Normal QTc (P-value $<0.05$ ); although LFM was higher in the prolonged QTc group, it was not significant $(\mathrm{P}$-value $=0.105)$. In females, AFM, LFM, TFM, and Total FM were more elevated in the normal QTc group, although they were in contrast to male results as they were not statistically significant ( $P$-value $>0.05)$. Also, none of the FFMs showed a considerable difference between QTc groups in both genders.

BMI did not show any significant difference between normal QTc and prolonged QTc interval groups in both genders (P-value $>0.05$ ). BMI was higher in the male prolonged QTc group than the male normal QTc group, but it was not significant $(\mathrm{P}$-value $=0.170)$. In females, higher BMI was observed in the normal QTc group, but it had not shown a significant difference between the $\mathrm{QTc}$ group $(\mathrm{P}$-value $=0.329)$. Also, the mean heart rate was $64.77 \pm 10.09 \mathrm{bpm}, 74.18 \pm 11.68 \mathrm{bpm}$ in normal and $73.69 \pm 13.61 \mathrm{bpm}, 77.48 \pm 12.09 \mathrm{bpm}$ in prolonged QTc group respectively in male and female, which was a significant difference $(\mathrm{P}$-value $<0.001)$.

In male, Arms $(B=0.158)$, Legs $(B=0.104)$, Trunk $(B=0.152)$ and total $(B=0.142) F M$ percentage showed significant positive relationship with continuous QTc (P-value $<0.001$ ). Also, FMI and FM/FFM ratio in total and all regions had significant positive associations with continuous QTc (P-value $<0.001)$. The highest beta-coefficient of FMI was related to trunk $(B=0.148)$, followed by total $(B=0.137)$. All of these relationships remained significant after multi-variable adjust in linear regression (P-value $<0.01)$. In logistic regression, after multivariable-adjusting, FM/FFM ratios in total, trunk, and arms showed significant associations with QTc $>450 \mathrm{~ms}$ with ORs 6.66, 5.58, and 11.31, respectively (P-value $<0.05$ ). In fat percentages, just arms and trunk showed significant associations (P-value $<0.05)$. All B coefficients, ORs, and their P-values are reported as the results of both unadjusted and multivariable-adjusted regression in Table 3 for males and Additional File 1: Table A1 for females.

Surprisingly, none of the body composition variables in females did show a significant correlation with continuous QTc. In logistic regression, only four ORs remained significant after multi-variable adjusted, related to total and truncal fat percentage and FM/FFM ratio. In contrast to male results, these ORs were lower than 1.00. FM/FFM ratios in total, and trunk showed significant associations with QTc $>450 \mathrm{~ms}$ with ORs $0.32(0.12-0.90)$, and 0.31 $(0.12-0.82)$ respectively $(\mathrm{P}$-value $<0.05)$. Figure 1 shows the linear regression line and 95\% CI between QTc interval and fat mass in different body parts in both genders.

Additional File 2 would show the results of this study if QT intervals were corrected by Fridericia's formula. Additional File 2: Table B1 has reported that none of the body fat composition data shows significant differences between QT interval groups in men. At the same time, the mean of fat percentage, FMI, and FM/FFM ratio in total, legs and trunk had significant differences between QT interval groups in women(P-value $<0.05)$. Regarding the association of Fridericia's formula corrected QT interval and body composition data in male subjects, Additional File 2: Table B1 shows a lower count of significant beta-coefficients and lower beta-coefficients values than the main results (using Bazett's formula). Also, there was not any significant OR related to men (Additional File 2: Table B2). In contrast, more significant ORs were observed in female subjects, and none of the beta-coefficients of linear regressions were significant. However, 
Table 2 The mean and standard deviation of total and regional body composition data and BMI in total, normal QTC and prolonged QTc interval groups according to gender

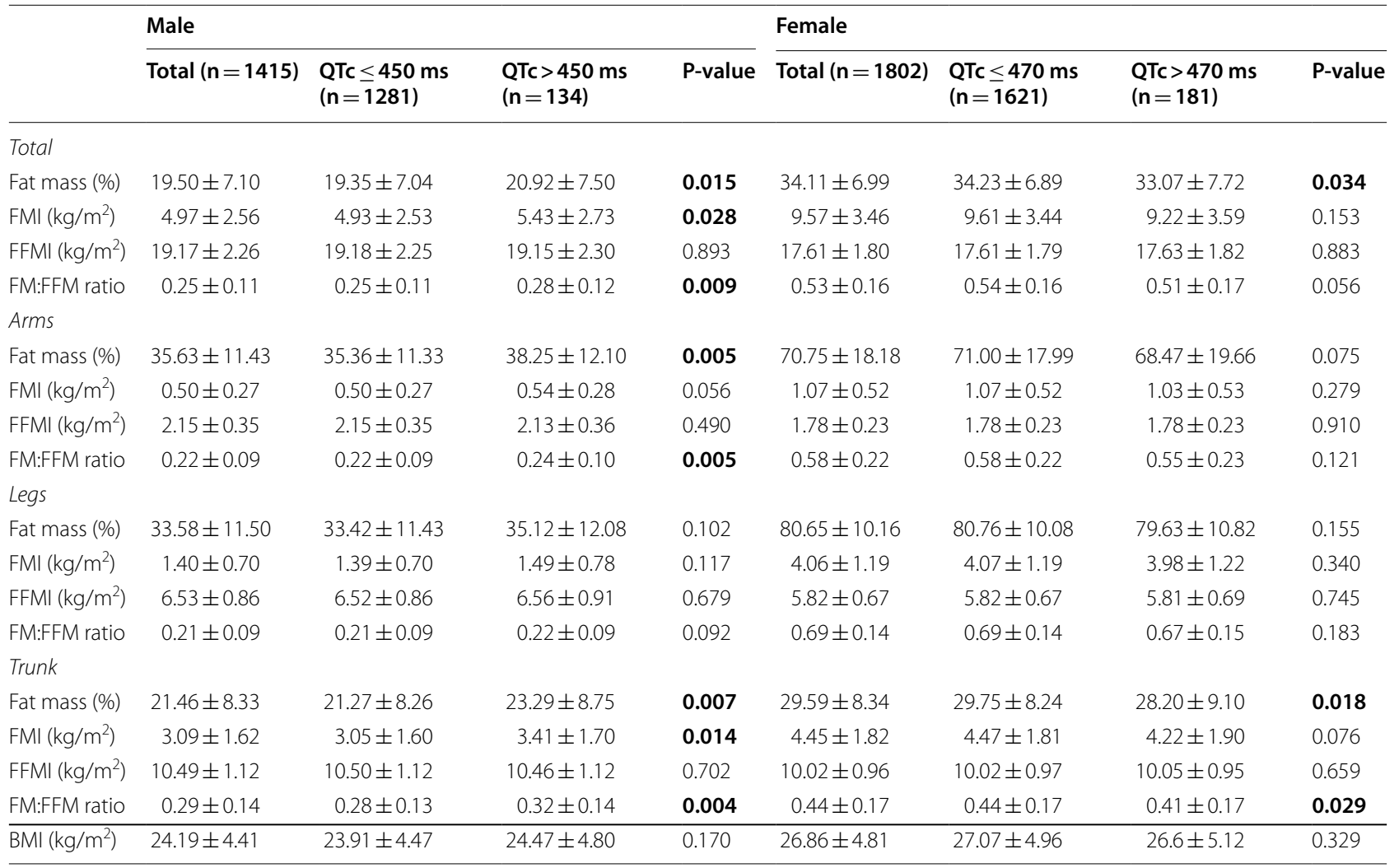

QTc = corrected QT interval by Bazett formula. P-value reported as the result of independent-samples t-test between normal and prolonged QTc interval groups. Statistically significant $\mathrm{P}$-values are bolded $(\mathrm{P}$-value $<0.05)$

both QT correction formulas showed positive and more than $1.00 \mathrm{OR}$ for males and negative and less than 1.00 OR for females (Additional File 2: Table B3).

\section{Discussion}

\section{Main findings}

This study suggests that (i) in male contributors, there were significantly higher FM/FFM ratio in total body, arms, and trunk in prolonged QTc group compared to normal QTc group. These body composition variables, as well as FM/FFM ratio in legs, showed unequal significant positive correlations with QTc. Also, FMI of total and all regional fat had a significant positive association with QTc. (ii) in female contributors, there were substantial differences in the mean of FM/FFM ratio in trunk and legs between normal and prolonged QTc groups. Surprisingly, the normal QTc group had higher body composition variables in women. Also, none of the body composition variables had a significant correlation with continuous QTc. (iii) Fat mass ratios in the trunk, arms, total, and legs, respectively, with a higher to a lower betacoefficient, were associated with QTc interval in males, and such associations were not observed in females.
These findings were related to a population without any electrolyte imbalance. These relations remained significant after multi-variable adjusting for age, smoking status, total cholesterol, high-density lipoprotein, and blood pressure, history of CHD, diabetes, and taking any medication that affects QT interval [25].

\section{Interpretation of data}

To our knowledge, this is the first study to seek if different patterns of anatomical fat distribution in the body could affect the QTc interval among people; that is why we used one of the most significant databases of ECG in an Iranian population. Our study extends the knowledge from previous studies in fat mass and QTc interval by showing which part of body fat mass was related to QTc interval. Few previous studies have focused on total fat percentage, visceral and subcutaneous fat depots, upper body obesity (waist to hip circumference ratio $\geq 0.85$ ), and BMI subjects [31-35]. It should be mentioned that the difference between the mean of BMI in normal and prolonged QTc interval groups was not significant in our study. Still, we were able to show body fat mass significant association with QTc interval in men even after 
Table 3 The association between total and regional body composition data and QTc interval in male

\begin{tabular}{|c|c|c|c|c|c|c|c|c|}
\hline & \multicolumn{4}{|c|}{ Unadjusted } & \multicolumn{4}{|c|}{ Multi-variable adjusted } \\
\hline & \multicolumn{2}{|c|}{ QTc (continuous) } & \multicolumn{2}{|l|}{$\mathrm{QTc}>450 \mathrm{~ms}$} & \multicolumn{2}{|c|}{ QTc (continuous) } & \multicolumn{2}{|l|}{$\mathrm{QTc}>450 \mathrm{~ms}$} \\
\hline & Beta & P-value & OR $(95 \% \mathrm{Cl})$ & P-value & Beta & P-value & OR $(95 \% \mathrm{Cl})$ & P-value \\
\hline \multicolumn{9}{|l|}{ Total } \\
\hline Fat mass (\%) & 0.142 & $<0.001$ & $1.03(1.01-1.06)$ & 0.015 & 0.125 & $<0.001$ & $1.03(1.00-1.06)$ & 0.057 \\
\hline $\mathrm{FMI}\left(\mathrm{kg} / \mathrm{m}^{2}\right)$ & 0.137 & $<0.001$ & $1.08(1.01-1.15)$ & 0.029 & 0.122 & $<0.001$ & $1.07(0.99-1.15)$ & 0.078 \\
\hline FFMI $\left(\mathrm{kg} / \mathrm{m}^{2}\right)$ & 0.038 & 0.154 & $1.00(0.92-1.08)$ & 0.893 & 0.029 & 0.331 & $0.99(0.91-1.09)$ & 0.885 \\
\hline FM:FFM ratio & 0.149 & $<0.001$ & $8.32(1.70-40.73)$ & 0.009 & 0.131 & $<0.001$ & $6.66(1.11-40.13)$ & 0.038 \\
\hline \multicolumn{9}{|l|}{ Arms } \\
\hline Fat mass (\%) & 0.158 & $<0.001$ & $1.02(1.01-1.04)$ & 0.005 & 0.145 & $<0.001$ & $1.02(1.00-1.04)$ & 0.018 \\
\hline $\mathrm{FMI}\left(\mathrm{kg} / \mathrm{m}^{2}\right)$ & 0.124 & $<0.001$ & $1.74(0.98-3.09)$ & 0.060 & 0.113 & $<0.001$ & $1.73(0.92-3.27)$ & 0.090 \\
\hline FFMI $\left(\mathrm{kg} / \mathrm{m}^{2}\right)$ & 0.008 & 0.773 & $0.84(0.50-1.39)$ & 0.490 & 0.007 & 0.813 & $0.88(0.49-1.57)$ & 0.659 \\
\hline FM:FFM ratio & 0.157 & $<0.001$ & $12.61(2.09-76.09)$ & 0.006 & 0.142 & $<0.001$ & $11.31(1.51-84.94)$ & 0.018 \\
\hline \multicolumn{9}{|l|}{ Legs } \\
\hline Fat mass (\%) & 0.104 & $<0.001$ & $1.01(1.00-1.03)$ & 0.102 & 0.076 & 0.0101 & $1.01(0.99-1.03)$ & 0.271 \\
\hline $\mathrm{FMI}\left(\mathrm{kg} / \mathrm{m}^{2}\right)$ & 0.107 & $<0.001$ & $1.21(0.95-1.54)$ & 0.117 & 0.090 & 0.002 & $1.19(0.91-1.56)$ & 0.202 \\
\hline FFMI $\left(\mathrm{kg} / \mathrm{m}^{2}\right)$ & 0.064 & 0.015 & $1.04(0.85-1.28)$ & 0.679 & 0.081 & 0.007 & $1.09(0.87-1.38)$ & 0.443 \\
\hline FM:FFM ratio & 0.106 & $<0.001$ & $5.55(0.76-40.64)$ & 0.092 & 0.079 & 0.008 & $3.87(0.40-37.84)$ & 0.245 \\
\hline \multicolumn{9}{|l|}{ Trunk } \\
\hline Fat mass (\%) & 0.152 & $<0.001$ & $1.03(1.01-1.05)$ & 0.008 & 0.137 & $<0.001$ & $1.03(1.00-1.05)$ & 0.032 \\
\hline FMI $\left(\mathrm{kg} / \mathrm{m}^{2}\right)$ & 0.148 & $<0.001$ & $1.14(1.03-1.27)$ & 0.014 & 0.132 & $<0.001$ & $1.12(1.00-1.27)$ & 0.053 \\
\hline FFMI $\left(\mathrm{kg} / \mathrm{m}^{2}\right)$ & 0.025 & 0.346 & $0.97(0.83-1.14)$ & 0.702 & -0.004 & 0.900 & $0.94(0.78-1.12)$ & 0.457 \\
\hline FM:FFM ratio & 0.160 & $<0.001$ & $6.76(1.84-24.79)$ & 0.004 & 0.145 & $<0.001$ & $5.58(1.31-23.72)$ & 0.020 \\
\hline
\end{tabular}

$\mathrm{B}=$ beta coefficient, $\mathrm{QTC}=$ Corrected QT interval by Bazett's formula, $\mathrm{OR}=\mathrm{Odds}$ ratio, $\mathrm{Cl}=$ Confidence interval. Statistically significant $\mathrm{P}$-values are bolded

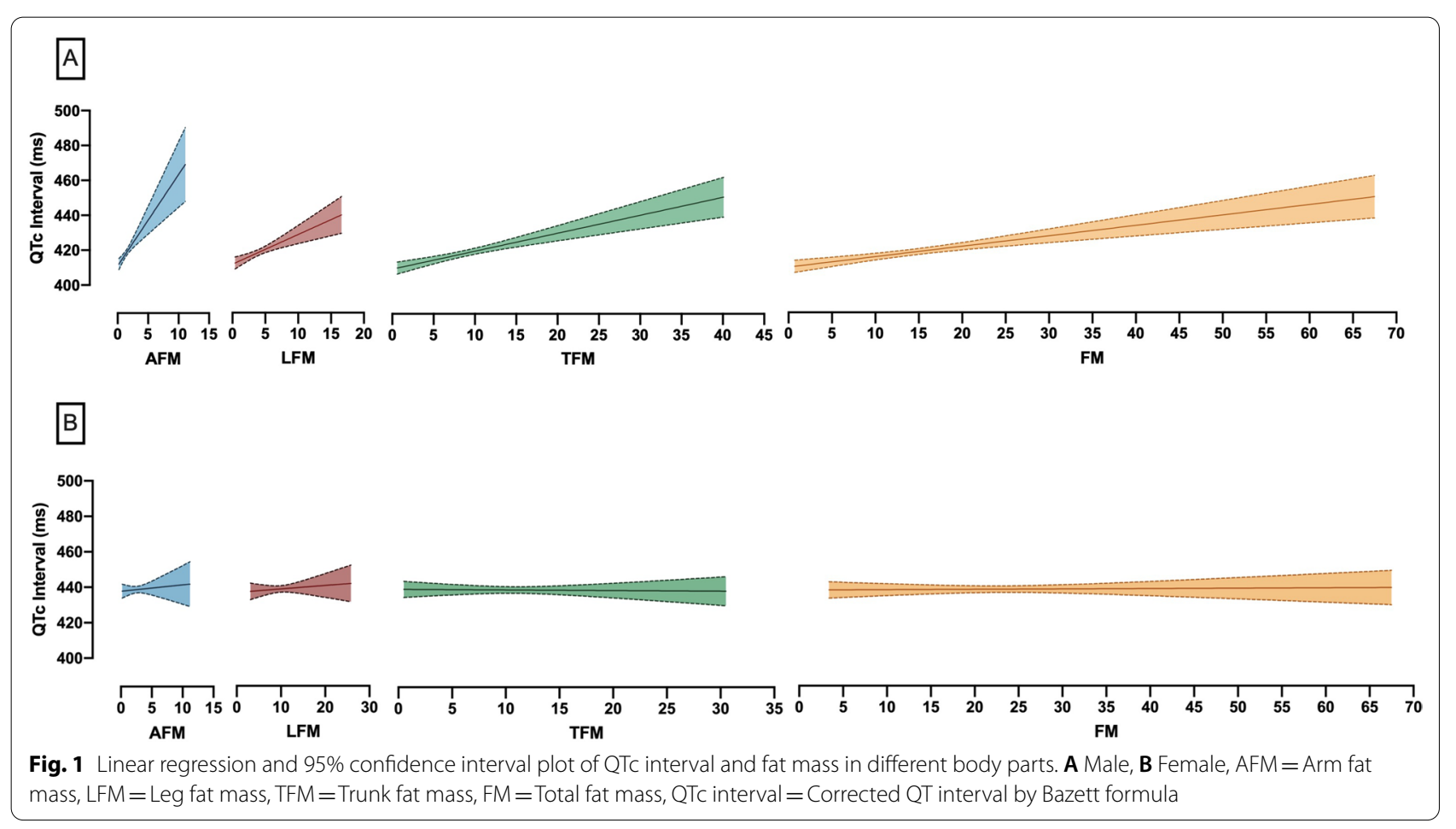


multi-variable adjusting. As our study is observational, we decided to minimize other variables that may affect body fat and QTc interval by adjusting for them. Age, BP, TC, HDL, current smoking status, history of CHD, DM, HTN, and using selected medications known for prolonging QTc were the adjusting variables. It is noticeable that every significant result remained significant after multi-variable adjusting, so it can interpret that body fat mass may be an "independent risk factor" for increasing QTc, apart from conventional CVD risk factors.

The coincidence of higher QTc intervals and obesity can have a supra additive adverse effect on health. On the other hand, these two can be interrelated. Previous studies observed prolonged QTc be related to obesity and its increase [36]. Also, Braschi et al.; reported that mean of corrected QT intervals in subjects with $\mathrm{BMI}>30$ is higher than $40 \mathrm{~ms}$ compared to subjects with $\mathrm{BMI}<25$ [35]. With a smaller sample size, El-Gamal et al. demonstrated that relative body fat is associated with QTc interval [31]. Hillerbrand et al [32] has suggested that more visceral fat accumulation is associated with prolongation of QTc. This study was based on the magnetic resonance imaging evolution of visceral fat. We reported the same results for body fat measures and QTc in men, but our study's advantage was measuring different body parts' fat mass. This advantage enabled us to report the correlation between the different anatomic distribution of fat and QTc interval more accurately. Although body fat and QTc did not represent a significant association in women in our study, Park et al [33] and Peiris et al [34] showed a positive correlation between QTc and body fat women. The first noticeable weak point for these two studies might be their petite sample sizes (31 and 27 participants, respectively). Moreover, the method of body fat measurements is different from our method. The former study method of measurement was based on Lohman et al. calculation formula, and the second study method measured body fat mass by hydrodensitometry. Another reason may be that the female subjects in our population reported a much worse cardiovascular profile, as it was addressed before [13]. For example, the frequency of CHD in females was $11.4 \%$ compared to $5.7 \%$ in male subjects. These differences in the baseline cardiovascular characteristic of the two groups may explain the contrary findings between the two genders.

The load-capacity model in this study was utilized to identify people with insufficient muscle mass and excessive adiposity, using a tiered risk approach. The loadcapacity model integrates the physiological impacts of adiposity and lean body mass components within the same individual to operationalize body composition assessment. The proportionate contribution of each component to physiological functions, which considers hormonal and age-related body composition changes, will determine risk [26]. The two indices' basic premise is that an individual's metabolic load is determined by their contribution relative to one another, not by a specific amount of the two separate components [37]. In other words, the issue is not about fat mass itself but the balance between lifestyle (with a high FM as a result of positive metabolic load) and functional capacity of the body [26]. Several previous studies have been evaluated the load-capacity model and worse health outcomes. Auyeung et al. [38] showed that FM/FFM ratio might predict a year increased risk of physical disability in the Chinese elderly. Also, it has been suggested the associations of the load-capacity model with blood pressure [39] and metabolic syndrome [40]. In this study, for the first time, the load-capacity model of total and different body regions is associated with QTc interval.

Comparing our data with previously published population reference values demonstrated that in all age groups, our population FM/FFM ratio values were lower than population reference values of FM/FFM ratio calculated by using both DXA [26] and BIA [41] method in both genders. Moreover, the illustration of 5th, 15th, 25th, 50th, 75th, 85th, and 95th percentiles of FM/FFM ratio in total and regional fat depots according to age have been presented in Figs. 2 and 3. In male subjects, the relationships were linear overall, which was in line with the previous literatures [26].

Several pathophysiological mechanisms could explain our findings. Upper body obesity has been reported as a risk factor for CHD, consequently prolonging the QTc interval [42]. Possible mechanisms of the prolonged QTc in obesity include autonomic nervous system alternation and repolarization abnormalities, increased cardiac output, secreted cytokines by adipose tissue, electrolyte abnormalities, increasing plasma free fatty acid (FFA) levels, insulin resistance, and hyperinsulinemia. Gao et al [43] reported that subjects with upper-body obesity had higher autonomic nervous system activity. Dominant sympathetic over parasympathetic activation leads to a higher heart rate and influences ventricular repolarization [44]. Alternation of autonomic nervous system balance can also be due to elevated stroke volume and cardiac output in obese patients [31]. Underlie of the association of obesity and fat tissue with the autonomic nervous system could be cytokine secretion by adipose tissue [45]. The secreted pro-inflammatory cytokines such as leptin can directly stimulate the central sympathetic nervous system [45-47]. As metabolic mechanisms, it can be demonstrated that prolonged QTc interval may be due to increased FFA [48, 49], insulin resistance, and hyperinsulinemia [34] in more obese individuals. Scherrer et al [50] suggested that body fat is an 

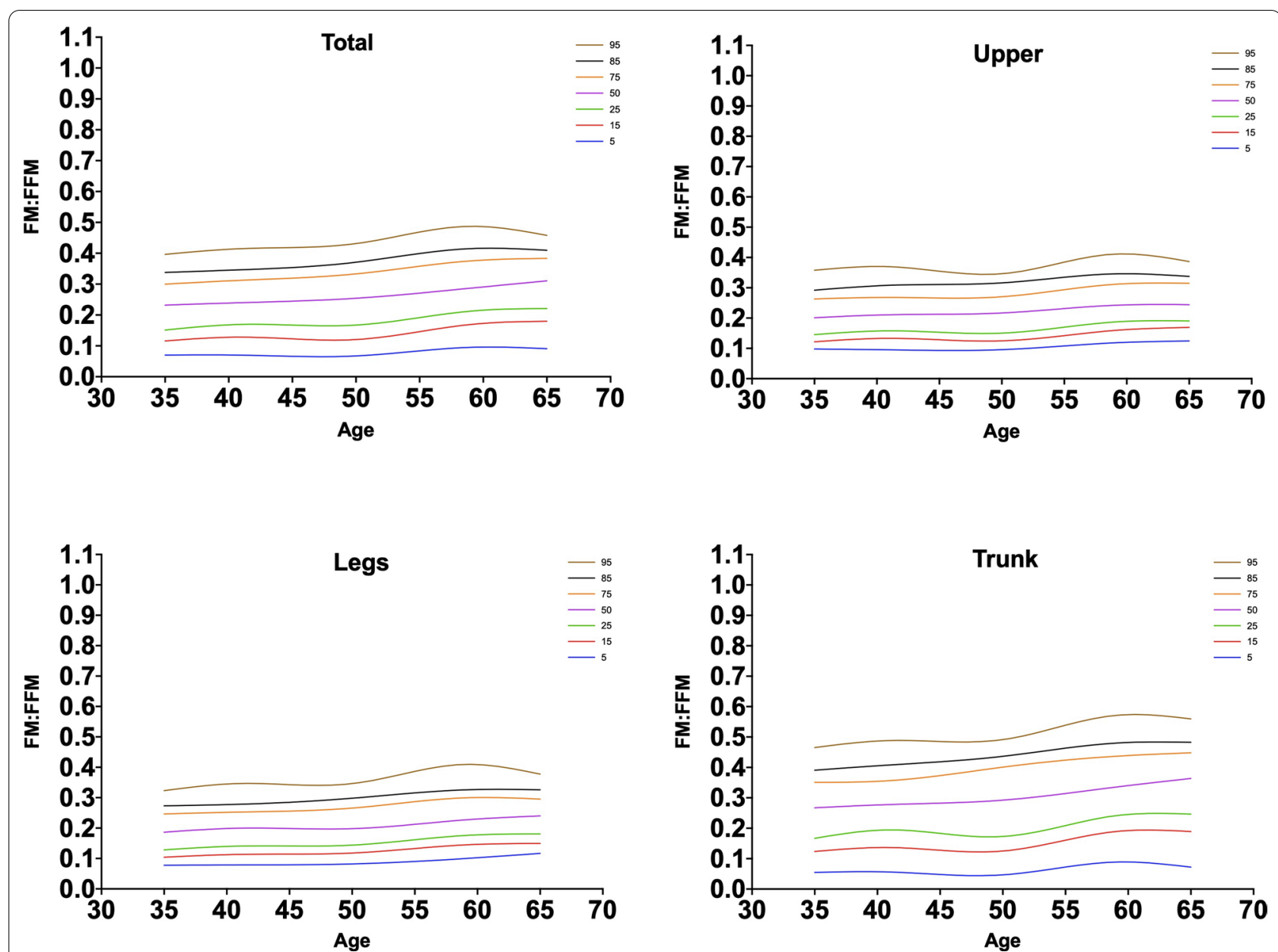

Fig. 2 Smoothed fat mass to fat-free mass ratio percentiles for men in total and different regional fat deposits. The percentiles shown are 5 th, 15 th, 25th, 50th, 75th, 85th, and 95th

indicator of muscle sympathetic nerve discharge, and it is in a positive correlation with fasting insulin level. Also, Behrasi et al [51] suggested that HOMA-IR is positively correlated with body fat mass as an indicator of insulin resistance. So, it can be interpreted that more fat tissue leads to more insulin resistance and hyperinsulinemia. Another underlying mechanism of hyperinsulinemia may be due to its ion exchange role at the myocardium, which may finally induce QTc prolongation.

After all, the BIA method provided us more details about the anatomic distribution of fat through the body. Although the body composition data of total and regional parts of the body were closely intercorrelated (Additional File 1: Table A2), it was suggested that body fat mass, including trunk, arms, and legs, may be related to QTc interval unevenly. The truncal fat mass ratio had the highest value of beta-coefficient among body composition values; it may be the deposited fat in the epicardium that plays a role in the repolarization. Logically higher truncal fat indicates a higher fat deposition on the epicardial surface of the heart. This may be the underlying mechanism of prolonged repolarization with possible electrical sequelae for the myocardium. Also, some previous studies in the case of epicardial adipose tissue and QT interval reported a significant relationship between these two [52, 53]. Moreover, pericardial adipose tissue was shown to be associated with impaired fasting plasma glucose $[18,54]$, insulin resistance, and hypertension $[19,55]$. These associations may show that pericardial adipose tissue can trigger the mechanisms mentioned above and prone subjects to CHD. Or maybe it is just a matter of ECG recording in participants with higher fat mass in the trunk? Perhaps more prolonged QT simply indicates a lag in voltage recording. This can only be measured utilizing more sophisticated devices that can record voltage changes pretty fast to exclude any delays produced by the higher fat. Of course, the extended follow-up data, when compiled, will tell us about the 

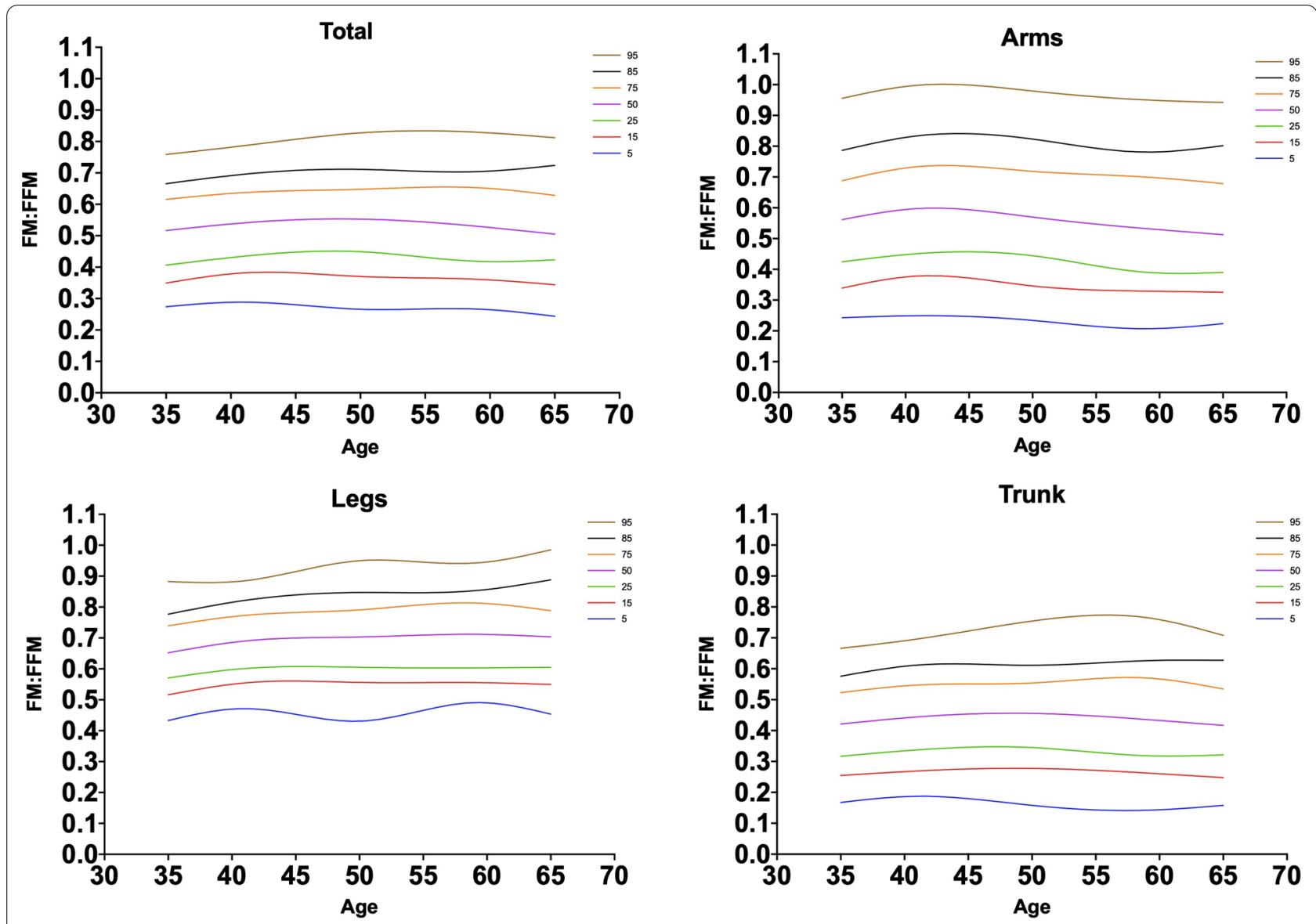

Fig. 3 Smoothed fat mass to fat-free mass ratio percentiles for women in total and different regional fat deposits. The percentiles shown are 5 th, 15th, 25th, 50th, 75th, 85th, and 95th

prognosis of this study's observations, and we will wait to see if there are higher CVD events in those with higher fat indices, mostly higher amount of FM/FFM ratios and QTc interval compared to other different body parts' fat mass. We also suggest that this association between fat mass and QTc should investigate different variables such as electrolyte level, insulin level, FFA, physical activity, and calorie intake to minimize their influence on this relationship.

\section{Strengths and limitations}

A strength in our study was our larger study population in comparison to other studies on this topic. Data collection of digital ECGs were done under highly standardized conditions and enabled us to report more precise ECG data. Another strength was the extensive measurements of confounding factors in the Fasa PERSIAN Cohort Study, which examined the relationship of QTc interval and fat mass apart from influential factors. Also, to our knowledge, this is the first study to seek if different patterns of anatomical fat distribution in the body have any association with the QTc interval among an Iranian population.

Our study was not free from limitations. First, as this study has a cross-sectional design, it limits our ability to infer any causality. Second, our study was based on a rural population within the age range of 35-70, which restricted us from extending our findings to a younger or urban community. Third, body composition was assessed with a BIA device. BIA systems are not considered reference methods for body composition analysis but as predictive methods with population-specific predictive algorithms. These potential errors in body composition analysis were an undeniable limitation.

At last, although the linear association of fat mass and QTc interval was strong enough, the association between fat mass and prolonged QTc interval (as a binary variable) showed a slight level of significance. So, it could not be interpreted that higher fat mass would lead to QTc intervals more than cut-points, which may be due to our small sample size in prolonged QTc interval groups. Regarding the last limitation, 
although the sample size of prolonged QTc interval in female subjects was reasonable compared to male subjects and $470 \mathrm{~ms}$ is a well-established cut point for females, another cut-point was used for female subjects in case $470 \mathrm{~ms}$ be a bit high (Additional File 3). Surprisingly, using a lower cut point on Bazett's formula corrected QT interval led to having zero significant difference between QT interval groups (Additional File 3: Table C1) and ORs (Additional File 3: Table C2). While using the same cut point on Fridericia's formula, corrected QT interval led to having more significant differences between QT interval groups (Additional File 3: Table C1) and ORs (Additional File 3: Table C2). These results may vary from the study's primary results, but in general, they were in line with the main results as the female subjects showed negative relationships between body composition data and QTc (even in another QT corrected formula and cut-point). For sure, longer follow-ups in our study, other and larger populations, can reveal more results in these relationships as life is more than calculating B-coefficients.

\section{Conclusion}

Our study suggested that FM/FFM ratios in trunk, arms, total, and legs were positively associated with QTc interval in males, respectively, from a higher to a lower betacoefficient. Our study implies that body fat mass and different body parts' fat mass may be independent risk factors for higher QTc interval and, consequently, more cardiovascular events, which should be investigated in further studies.

\section{Supplementary Information}

The online version contains supplementary material available at https://doi. org/10.1186/s12872-021-02095-2.

Additional file 1. The association between QTC interval and body fat composition data in female and the intercorrelation of total and regional body fat composition data in both genders.

Additional file 2. The mean of total and regional body composition data and BMI in QT interval groups and the association between QT interval, total and regional body fat composition, and fat mass index in both genders using Fridericia's formula for correcting QT intervals.

Additional file 3. The mean of total and regional body composition data in QTc interval groups and the association between QTc interval, total and regional body fat composition, and fat mass index in female subjects using a lower cut-point (> $460 \mathrm{~ms}$ ).

\section{Acknowledgements}

The authors appreciate Kosar Hashemzadeh for her assistance on manuscript draft preparations and Dr. Alireza Shamloo Sepehri for his grateful comments on this study.

\section{Authors' contributions}

Conceptualization: MHY, MF. Methodology: MHY, MF, MMN. Software: MHY, MMN. Validation: MM, SD. Formal analysis: MHY, MMN. Investigation: MHY, SD.
Resources: EB, MF. Data curation: SD, MM. Writing (original draft preparation): MHY, MF. Writing (review and editing): EB, MF. Visualization: EB, MF. Supervision: MF. Project administration: MF. Funding acquisition: MF. All authors read and approved the final manuscript.

\section{Funding}

This research did not receive any specific grant from funding agencies in the public, commercial, or not-for-profit sectors.

\section{Availability of data and materials}

The datasets used and/or analyzed during the current study are available from the corresponding author on reasonable request to the corresponding author.

\section{Declarations}

\section{Ethics approval and consent to participate}

The participants were informed about the research objectives, and the written informed consent was obtained from the subjects before starting the survey. Our study was designed by following the relevant guidelines and regulations of our regional and national research ethics committees. Also, the protocol of this study has the approval of regional and national research ethics committees (the equivalent of institutional review boards) of FUMS (reference number: IR.FUMS.REC.1397.036).

\section{Consent for publication}

Not applicable.

\section{Competing Interests}

The authors declare no conflicts of interest in this study.

\section{Author details}

${ }^{1}$ Student Research Committee, Fasa University of Medical Sciences, Fasa, Iran. ${ }^{2}$ Noncommunicable Diseases Research Center, Fasa University of Medical Sciences, Fasa, Iran. ${ }^{3}$ Non-communicable Diseases Research Center, Fars Heart Foundation, Kowsar Hospital, Shiraz, Iran.

Received: 22 February 2021 Accepted: 1 June 2021

Published online: 05 June 2021

\section{References}

1. Benoit SR, Mendelsohn AB, Nourjah P, et al. Risk factors for prolonged QTc among US adults: third National Health and Nutrition Examination Survey. Eur J Cardiovasc Prev Rehabil. 2005;12(4):363-8.

2. Akylbekova EL, Crow RS, Johnson WD, et al. Clinical correlates and heritability of QT interval duration in blacks: the Jackson Heart Study. Circ Arrhythm Electrophysiol. 2009;2(4):427-32.

3. Sohaib SMA, Papacosta O, Morris RW, et al. Length of the QT interval: determinants and prognostic implications in a population-based prospective study of older men. J Electrocardiol. 2008;41(6):704-10.

4. Castro VM, Clements CC, Murphy SN, et al. QT interval and antidepressant use: a cross sectional study of electronic health records. BMJ. 2013;346:f288.

5. Tisdale JE, Jaynes HA, Kingery JR, et al. Development and validation of a risk score to predict QT interval prolongation in hospitalized patients. Circ Cardiovasc Qual Outcomes. 2013;6(4):479-87.

6. Yazdanpanah MH, Sayyadipoor S, Hojati SR, et al. The association of metabolic syndrome and its components with electrocardiogram parameters and abnormalities among an iranian rural population: the Fasa PERSIAN Cohort Study. Diabetes Metab Syndr Obes Targets Ther. 2020;13:2975.

7. Pickham D, Helfenbein E, Shinn JA, et al. High prevalence of corrected QT interval prolongation in acutely ill patients is associated with mortality: results of the QT in Practice (QTIP) Study. Crit Care Med. 2012;40(2):394-9.

8. Pasquier $\mathrm{M}$, Pantet $\mathrm{O}, \mathrm{Hugli} \mathrm{O}$, et al. Prevalence and determinants of QT interval prolongation in medical inpatients. Intern Med J. 2012;42(8):933-40.

9. Grandinetti A, Seifried S, Mor J, et al. Prevalence and risk factors for prolonged QTc in a multiethnic cohort in rural Hawaii. Clin Biochem. 2005;38(2):116-22. 
10. Roden DM. Drug-induced prolongation of the QT interval. N Engl J Med. 2004;350(10):1013-22.

11. Drenick EJ, Fisler JS. Sudden cardiac arrest in morbidly obese surgical patients unexplained after autopsy. Am J Surg. 1988;155(6):720-6.

12. Electrocardiographic observations before, during and after rapid weightloss in morbidly obese subjects. Circulation; 1981. Amer Heart Assoc 7272 Greenville Avenue, Dallas, TX 75231-4596.

13. Yazdanpanah MH, Homayounfar R, Khademi A, et al. Short sleep is associated with higher prevalence and increased predicted risk of cardiovascular diseases in an Iranian population: Fasa PERSIAN Cohort Study. Sci Rep. 2020;10(1):1-13.

14. Kazempour-Ardebili S, Ramezankhani A, Eslami A, et al. Metabolic mediators of the impact of general and central adiposity measures on cardiovascular disease and mortality risks in older adults: Tehran Lipid and Glucose Study. Geriatr Gerontol Int. 2017;17(11):2017-24.

15. Hubert HB, Feinleib M, McNamara PM, et al. Obesity as an independent risk factor for cardiovascular disease: a 26-year follow-up of participants in the Framingham Heart Study. Circulation. 1983;67(5):968-77.

16. Omran J, Bostick BP, Chan AK, et al. Obesity and ventricular repolarization: a comprehensive review. Prog Cardiovasc Dis. 2018;61(2):124-35.

17. Carella M, Mantz S, Rovner D, et al. Obesity, adiposity, and lengthening of the QT interval: improvement after weight loss. Int J Obes Relat Metab Disord. 1996;20(10):938-42.

18. Iozzo P, Lautamaki R, Borra R, et al. Contribution of glucose tolerance and gender to cardiac adiposity. J Clin Endocrinol Metab. 2009;94(11):4472-82.

19. Sharma AM. Mediastinal fat, insulin resistance, and hypertension: Am Heart Assoc, 2004.

20. Polanczyk CA, Rohde LE, Moraes RS, et al. Sympathetic nervous system representation in time and frequency domain indices of heart rate variability. Eur J Appl Physiol Occup Physiol. 1998;79(1):69-73.

21. Napolitano C, Schwartz PJ, Brown AM, et al. Evidence for a cardiac ion channel mutation underlying drug-induced QT prolongation and lifethreatening arrhythmias. J Cardiovasc Electrophysiol. 2000;1 1(6):691-6.

22. Day CP, McComb JM, Campbell R. QT dispersion: an indication of arrhythmia risk in patients with long QT intervals. Heart. 1990;63(6):342-4.

23. Ludomirsky A, Klein HO, Sarelli P, et al. QT prolongation and polymorphous ("torsade de pointes") ventricular arrhythmias associated with organophosphorus insecticide poisoning. Am J Cardiol. 1982;49(7):1654-8.

24. Farjam M, Bahrami H, Bahramali E, et al. A cohort study protocol to analyze the predisposing factors to common chronic non-communicable diseases in rural areas: Fasa Cohort Study. BMC Public Health. 2016;16(1):1090.

25. Woosley R, Heise, CW and Romero, KA. www.Crediblemeds.org; [Available from: www.Crediblemeds.org. Accessed 19 Sept 2018.

26. Siervo M, Prado CM, Mire E, et al. Body composition indices of a loadcapacity model: gender-and BMI-specific reference curves. Public Health Nutr. 2015;18(7):1245-54.

27. CARDIAX computerized ECG system: IMED Ltd. http://www.imed.hu/en html. Accessed 10 Jun 2020

28. Bazett HC. An analysis of the time relations of electrocardiograms. Heart. 1920;7:353-70

29. Vandenberk B, Vandael E, Robyns T, et al. Which QT correction formulae to use for QT monitoring? J Am Heart Assoc. 2016;5(6):e003264.

30. Moss AJ. Measurement of the QT interval and the risk associated with QTC interval prolongation: a review. Am J Cardiol. 1993;72(6):B23-5.

31. El-Gamal A, Gallagher D, Nawras A, et al. Effects of obesity on QT, RR, and QTc intervals. Am J Cardiol. 1995;75(14):956-9.

32. Hillebrand S, de Mutsert R, Christen T, et al. Body fat, especially visceral fat, is associated with electrocardiographic measures of sympathetic activation. Obesity. 2014;22(6):1553-9.

33. Park J, Swan P. Effect of obesity and regional adiposity on the QTc interval in women. Int J Obes. 1997:21(12):1104.
34. Peiris A, Thakur R, Sothmann M, et al. Relationship of regional fat distribution and obesity to electrocardiographic parameters in healthy premenopausal women. South Med J. 1991;84(8):961-5.

35. Braschi A, Abrignani MG, Francavilla VC, et al. Novel electrocardiographic parameters of altered repolarization in uncomplicated overweight and obesity. Obesity. 2011;19(4):875-81.

36. Frank S, Colliver JA, Frank A. The electrocardiogram in obesity: statistical analysis of 1,029 patients. J Am Coll Cardiol. 1986;7(2):295-9.

37. Wells JC. The thrifty phenotype: an adaptation in growth or metabolism? Am J Hum Biol. 2011;23(1):65-75.

38. Auyeung TW, Lee JSW, Leung J, et al. Adiposity to muscle ratio predicts incident physical limitation in a cohort of 3,153 older adults - an alternative measurement of sarcopenia and sarcopenic obesity. Age. 2013;35(4):1377-85.

39. Grijalva-Eternod CS, Lawlor DA, Wells JC. Testing a capacity-load model for hypertension: disentangling early and late growth effects on childhood blood pressure in a prospective birth cohort. PLOS ONE. 2013:8(2):e56078.

40. Lim K, Yang S, Kim T, et al. The association between the ratio of visceral fat to thigh muscle area and metabolic syndrome: the Korean Sarcopenic Obesity Study (KSOS). Clin Endocrinol (Oxf). 2010;73(5):588-94.

41. Xiao J, Purcell S, Prado C, et al. Fat mass to fat-free mass ratio reference values from NHANES III using bioelectrical impedance analysis. Clin Nutr. 2018;37(6):2284-7.

42. Moss AJ. Prolonged QT-interval syndromes. JAMA. 1986;256(21):2985-7.

43. Gao YY, Lovejoy JC, Sparti A, et al. Autonomic activity assessed by heart rate spectral analysis varies with fat distribution in obese women. Obes Res. 1996;4(1):55-63.

44. Abildskov J. Neural mechanisms involved in the regulation of ventricular repolarization. Eur Heart J. 1985;6(suppl_D):31-9.

45. Smith MM, Minson CT. Obesity and adipokines: effects on sympathetic overactivity. J Physiol. 2012;590(8):1787-801.

46. Ahima R, Osei SY. Leptin signaling. Physiol Behav. 2004;81:223-41.

47. He G, Pedersen SB, Bruun J, et al. Differences in plasminogen activator inhibitor 1 in subcutaneous versus omental adipose tissue in non-obese and obese subjects. Horm Metab Res. 2003:35(03):178-82.

48. Abraham R, Riemersma RA, Wood D, et al. Adipose fatty acid composition and the risk of serious ventricular arrhythmias in acute myocardial infarction. Am J Cardiol. 1989;63(5):269-72.

49. Corbi GM, Carbone S, Ziccardi P, et al. FFAs and QT intervals in obese women with visceral adiposity: effects of sustained weight loss over 1 year. J Clin Endocrinol Metab. 2002;87(5):2080-3.

50. Scherrer U, Randin D, Tappy L, et al. Body fat and sympathetic nerve activity in healthy subjects. Circulation. 1994;89(6):2634-40.

51. Behrasi F, Vadjdi S, Jeihooni A, et al. Some affecting factors of insulin resistance: do amounts of adipose tissue and age have independent impact on the development of insulin resistance? J Adv Pharm Educ Res. 2019;8:1-6.

52. Quisi A, Şentürk SE, Harbalıoğlu H, et al. The relationship between echocardiographic epicardial adipose tissue, P-wave dispersion, and corrected QT interval. Turk Kardiyol Dern Ars. 2018:46(6):471-8.

53. Kaplan O, Kurtoglu E, Nar G, et al. Evaluation of electrocardiographic T-peak to T-end interval in subjects with increased epicardial fat tissue thickness. Arq Bras Cardiol. 2015;105(6):566-72.

54. Perseghin G, Lattuada G, De Cobelli F, et al. Increased mediastinal fat and impaired left ventricular energy metabolism in young men with newly found fatty liver. Hepatology. 2008;47(1):51-8.

55. Bosy-Westphal A, Kossel E, Goele K, et al. Association of pericardial fat with liver fat and insulin sensitivity after diet-induced weight loss in overweight women. Obesity. 2010;18(11):2111-7.

\section{Publisher's Note}

Springer Nature remains neutral with regard to jurisdictional claims in published maps and institutional affiliations. 